ISSN 2072-6694

www.mdpi.com/journal/cancers

Review

\title{
HEXIM1, a New Player in the p53 Pathway
}

Qiao Jing Lew ${ }^{1}$, Kai Ling Chu ${ }^{1}$, Yi Ling Chia ${ }^{1}$, Nge Cheong ${ }^{1}$ and Sheng-Hao Chao ${ }^{1,2, *}$

1 Expression Engineering Group, Bioprocessing Technology Institute, A*STAR (Agency for Science, Technology and Research), 20 Biopolis Way, \#06-01, Singapore 138668, Singapore

2 Department of Microbiology, National University of Singapore, Singapore 117597, Singapore

* Author to whom correspondence should be addressed; E-Mail: jimmy_chao@bti.a-star.edu.sg; Tel: +65-6407-0899; Fax: +65-6478-9561.

Received: 27 May 2013; in revised form: 24 June 2013 / Accepted: 24 June 2013 /

Published: 4 July 2013

Abstract: Hexamethylene bisacetamide-inducible protein 1 (HEXIM1) is best known as the inhibitor of positive transcription elongation factor $\mathrm{b}(\mathrm{P}-\mathrm{TEFb})$, which controls transcription elongation of RNA polymerase II and Tat transactivation of human immunodeficiency virus. Besides P-TEFb, several proteins have been identified as HEXIM1 binding proteins. It is noteworthy that more than half of the HEXIM1 binding partners are involved in cancers. P53 and two key regulators of the p53 pathway, nucleophosmin (NPM) and human double minute-2 protein (HDM2), are among the factors identified. This review will focus on the functional importance of the interactions between HEXIM1 and p53/NPM/HDM2. NPM and the cytoplasmic mutant of NPM, NPMc+, were found to regulate P-TEFb activity and RNA polymerase II transcription through the interaction with HEXIM1. Importantly, more than one-third of acute myeloid leukemia (AML) patients carry $\mathrm{NPMc}+$, suggesting the involvement of HEXIM1 in tumorigenesis of AML. HDM2 was found to ubiquitinate HEXIM1. The HDM2-mediated ubiquitination of HEXIM1 did not lead to protein degradation of HEXIM1 but enhanced its inhibitory activity on P-TEFb. Recently, HEXIM1 was identified as a novel positive regulator of p53. HEXIM1 prevented p53 ubiquitination by competing with HDM2 in binding to p53. Taken together, the new evidence suggests a role of HEXIM1 in regulating the p53 pathway and tumorigenesis.

Keywords: HEXIM1; P-TEFb; p53; HDM2; NPM; NPMc+ 


\section{Introduction}

Hexamethylene bisacetamide-inducible protein 1 (HEXIM1) was initially identified in 1999 by Kusuhara, et al. from vascular smooth muscle cells treated with hexamethylene bisacetamide (HMBA), an inhibitor of proliferation [1]. In the same year, Ghatpande, et al. cloned the HEXIM1 cDNA from the presumptive heart-forming regions of chicken embryos and named it cardiac lineage protein-1 (CLP-1) [2]. The HEXIM1/CLP-1 knockout mice were embryonic-lethal and exhibited phenotypes of cardiac hypertrophy [3,4]. HEXIM1 was also identified as a binding protein of estrogen receptor $\alpha(E R \alpha)$ from a yeast two-hybrid screen using a MCF7 breast cancer cell cDNA library [5]. Estrogen was found to down-regulate HEXIM1 expression at both protein and mRNA levels. Because of this observation, HEXIM1 was also named as estrogen down-regulated gene 1 (EDG1) [5]. In 2003, research groups led by Olivier Bensaude and Qiang Zhou revealed a major biological function of HEXIM1. They demonstrated that HEXIM1 associated with positive transcription elongation factor $b$ (P-TEFb) and inhibited its activity [6,7].

$\mathrm{P}-\mathrm{TEFb}$ was identified and purified by David Price's group based on its sensitivity to 5,6-dichloro1-beta-D-ribofuranosylbenzimidazole (DRB), which inhibited RNA polymerase II (RNAP II) transcription at the elongation stage $[8,9]$. $\mathrm{P}-\mathrm{TEFb}$ is a protein complex composed of cyclin-dependent kinase 9 (CDK9) and a cyclin partner (i.e., cyclin T1, T2a, T2b, or K) with cyclin T1 being the predominant CDK9-associated cyclin [9-11]. P-TEFb phosphorylates the $C$-terminal domain of the largest subunit of RNAP II and allows the polymerase to enter the elongation phase $[9,12,13]$. Treatment of cells with flavopiridol, most potent and selective P-TEFb inhibiting compound, blocked $60-70 \%$ of RNAP II transcription as detected by nuclear run-on assays [14,15]. This pivotal finding clearly demonstrates that most of cellular genes are regulated by $\mathrm{P}-\mathrm{TEFb}$ at the elongation stage. Furthermore, three genome-wide studies using ChIP-on-chip assays found that RNAP II occupied the promoters of most protein-coding genes in Drosophila and human embryonic stem cells without entering into productive elongation [16-18]. Such genomic distribution of poised RNAP II molecules re-confirms the significance of $\mathrm{P}-\mathrm{TEFb}$ in gene expression. Transcription of many viruses is also under the control of P-TEFb. The best-studied regulation of viral transcription is Tat transactivation of human immunodeficiency virus (HIV). The HIV transactivator, Tat, recruits $\mathrm{P}-\mathrm{TEFb}$ to the viral promoter through the interaction with cyclin $\mathrm{T} 1$, resulting in the generation of full-length viral transcripts [19,20]. A compound screening was carried out in search for the inhibitors of HIV Tat transactivation. Surprisingly, all the compounds identified were P-TEFb inhibitors, indicating an essential role of $\mathrm{P}-\mathrm{TEFb}$ in controlling HIV transcription [21].

Having an estimated molecular mass of $150 \mathrm{kD}$, the $\mathrm{P}-\mathrm{TEFb}$ complex consisting of CDK9/cyclin T1 was shown to exhibit kinase activity [9]. It was later reported by several groups that the CDK9-containing protein complex with a larger molecular mass was isolated through glycerol gradient sedimentation, shedding lights that two different forms of P-TEFb existed in cells [22,23]. Initially, it was unknown what caused the enzymatic inhibition of P-TEFb within the large complex [24,25]. Soon after, both 7SK small nuclear RNA (snRNA) and HEXIM1 were identified and established as the new subunits of the large P-TEFb complex [6,7,24,25]. The 7SK snRNA-bound HEXIM1 exerted an inhibitory function on P-TEFb, while neither 7SK nor HEXIM1 alone instigate any effects [7,26]. It has been proposed that association with 7SK snRNA induces the conformational change of HEXIM1 protein 
and makes the cyclin T binding domain of HEXIM1 more accessible for P-TEFb binding [26]. In addition, a methylphosphate capping enzyme MEPCE and a La related protein LARP7 were identified as 7SK snRNA binding proteins [27-29]. A model for the regulatory mechanism of the P-TEFb protein complexes by HEXIM1 is summarized in Figure 1.

Figure 1. Two P-TEFb complexes are found in cells. The small P-TEFb complex, composed of cyclin $\mathrm{T} 1$ and $\mathrm{CDK} 9$, is the active form of $\mathrm{P}-\mathrm{TEFb}$. The kinase activity of $\mathrm{P}-\mathrm{TEFb}$ is inhibited when P-TEFb interacts with HEXIM1 and 7SK snRNA to form the large P-TEFb complex. Two other components of the large complex, MEPCE and LARP7, have been recently identified.

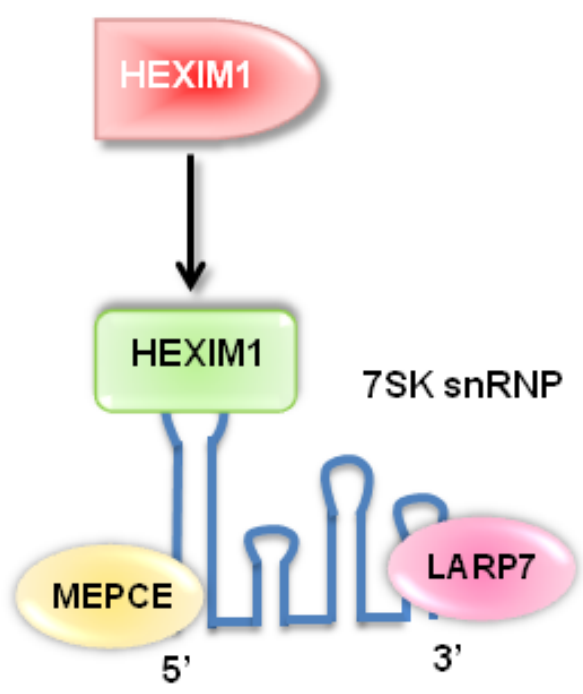

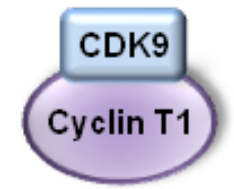

Small P-TEFb

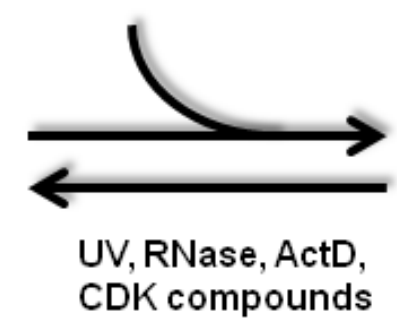

UV, RNase, ActD,
CDK compounds

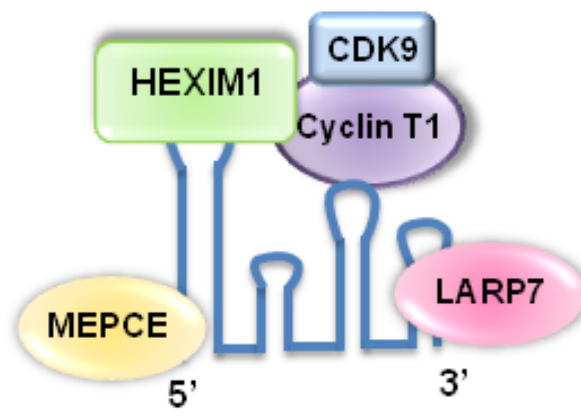

Large P-TEFb

HEXIM1 contains several functional domains. The $N$-terminus of HEXIM1, amino acids (a.a.) 1-150, has been characterized as a self-inhibitory domain (ID). Deletion of the ID enhances the inhibitory effects of HEXIM1 on P-TEFb activity [7,30]. The region between a.a. 150-180 of HEXIM1, which includes a stretch of basic residues, is referred to as the basic region (BR). The BR contains the binding motif for 7SK snRNA, KHRR (a.a. 152-155). When the KHRR sequence is replaced by ILAA, the mutant HEXIM1 protein fails to interact with 7SK snRNA and the formation of the large P-TEFb complex is disrupted [26]. The P-TEFb binding motif, PYNT (a.a. 202-205), is located between the $\mathrm{BR}$ and acidic region (AR, a.a. 210-250). In the absence of 7SK snRNA, the AR can interact with the adjacent BR. Since the P-TEFb binding motif is located between the BR and AR, the BR-AR interaction may establish an auto-inhibitory conformation which prevents the association between HEXIM1 and P-TEFb [31]. When 7SK snRNA binds to the BR, the BR-AR interaction is disrupted 
and the PYNT motif becomes accessible for P-TEFb binding [31]. HEXIM1 can form a homodimer or a heterodimer with a HEXIM1-related protein, HEXIM2, through the dimerization domain (DD) at the $C$-terminus of HEXIM1 [30,32,33].

Besides P-TEFb, several HEXIM1 binding proteins have been identified. HEXIM1 binds to histone deacetylases (HDACs) along with MyoD, indicating a role in regulating skeletal muscle cell differentiation [34]. Interaction between Importin $\alpha$, HEXIM1, and cyclin T1 has been reported. This finding suggests a possible mechanism for HEXIM1/Importin $\alpha$-mediated nucleo-cytoplasmic transport of cyclin T1 since no nuclear localization signals are present in cyclin T1 [35]. ER $\alpha$ is present in more than half of breast tumors and therefore, this receptor has been the most widely targeted protein in breast cancer therapy [36,37]. HEXIM1 competes with cyclin T1 in binding to ER $\alpha$. When associated with HEXIM1, the transcriptional activity of ER $\alpha$ is inhibited, suggesting a role of HEXIM1 in breast cancer [38]. It has been shown that HEXIM1 directly interacts with the p65 subunit of NF- $\mathrm{BB}$ and

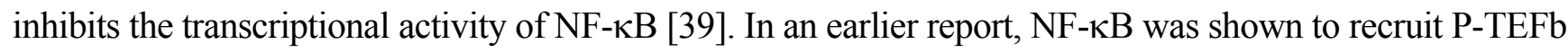
through the interaction with the p65 subunit, resulting in activation of NF-kB-dependent transcription [40]. These three-way interactions create an inevitable competition between HEXIM1 and P-TEFb in regulating the activity of NF-кB. Shimizu et al., demonstrated that HEXIM1 associated with the glucocorticoid receptor (GR) in the absence of 7SK snRNA and P-TEFb and regulated the GR-mediated gene expression [41]. The significance of this study is to reveal the involvement of HEXIM1 in the $\mathrm{P}-\mathrm{TEFb}$-independent bioprocesses.

Our recent studies demonstrated the functional correlation between HEXIM1 and the p53 signaling pathway. We identified p53 as well as two important regulators of p53, nucleophosmin (NPM) and human double minute-2 protein (HDM2), as the novel HEXIM1 binding proteins. In this review, we will summarize our findings and discuss the role of HEXIM1 in cancer.

\section{2. p53 and Its Regulators, HDM2 and NPM}

p53 is a tumor suppressor protein which regulates cell cycle and prevents cancer genesis. As such, p53 has been described as "the guardian of the genome" because of its role in conserving stability by preventing genome mutation [42]. The human p53 protein consists of 393 amino acids with five major functional domains: transactivation (TA), proline-rich (PR), DNA-binding (DBD), oligomerization (OLI), and negative regulation (NEG) domains [43].

The $N$-terminal TA domain, containing amino acids (a.a.) 1-42, recruits the basal transcriptional machinery, such as the TATA box binding protein (TBP) and TBP-associated factors, to activate the expression of p53 target genes [44,45]. The TA domain is followed by the PR region (a.a. 63-97), which is required for p53-mediated apoptosis and suppressing tumour cell growth [46,47]. The sequence-specific DBD is located within the central part of p53 (a.a. 102-292). Most mutations that deactivate p53 in cancer usually occur within the DBD and destroy the ability of p53 binding to its target DNA sequences [48]. The tetramerization of p53 takes place in the OLI domain (a.a. 323-356). Beside its importance for DNA binding, the OLI domain is also responsible for protein-protein interactions, post-translational modifications, and protein degradation of p53 [49]. Likewise, the NEG domain located at the $C$-terminus of p53 (a.a. 360-393) is involved in its own degradation. Holding major ubiquitination sites for HDM2, the ubiquitinated p53 is directed to proteosomal degradation [50-55]. 
Many regulators of p53 have been identified. Here we only focus on HDM2 and NPM, two HEXIM1 binding proteins. HDM2 (or MDM2, the mouse homolog), the best-known p53 regulator, is an E3 ubiquitin ligase that targets itself and p53 for protein degradation by the proteasome [56,57]. On the contrary, NPM functions as a positive regulator of p53 in the ARF-dependent and -independent manners. ARF, a tumor suppressor, binds to MDM2 and promotes rapid degradation of MDM2, resulting in p53 stabilization and accumulation [58]. In the ARF-dependent pathway, NPM associates with ARF in high-molecular-weight complexes. NPM stabilizes ARF by retarding its turnover and leads to p53 activation [59,60]. NPM is also found to interact with HDM2 directly and protect p53 from the HDM2-mediated degradation in an ARF-independent fashion [61]. Interestingly, both HDM2 and NPM are also involved in regulation of P-TEFb activity through modulating HEXIM1. In the following chapters, we will describe the involvement of NPM and HDM2 in regulating p53 in greater detail and discuss the functional interactions between NPM, HDM2, and P-TEFb/HEXIM1.

\section{NPM and NPMc+ Regulate P-TEFb Activity through the Interaction with HEXIM1}

NPM (also known as B23, numatrin, or NO38) encoded by the NPM1 gene is an abundant multifunctional phosphoprotein that mainly resides in nucleoli. It is required for several cellular processes such as ribosome biogenesis, cell proliferation, and transformation [62-64]. Apart from functioning as a histone chaperone protein in the formation of nucleosome, NPM is also involved in centrosome duplication $[65,66]$.

The connection of NPM to cancer and the p53 pathway has extensively been demonstrated. However, it is still debatable whether NPM function as a tumor suppressor or an oncogene. As mentioned earlier, NPM not only stabilizes p53 through antagonizing HDM2 [61], but also associates and stabilizes ARF within the nucleolus, resulting in induction of p53 [67,68]. Such positive regulation of p53 has been depicted in UV-exposed cells where NPM gets up-regulated and transiently translocated from nucleolus to nucleoplasm where it interacts with HDM2 [69]. Moreover, NPM also interacts directly with $\mathrm{p} 53$ to enhance the stability and transcriptional activation of p53 [70]. NPM is haploinsufficient for its function which denotes $N P M^{+-}$cells to have a significant degree of genomic instability, resulting in an increased susceptibility to oncogene transformation [71]. The tumor suppressing function of NPM is suggested based on these data. On the contrary, the elevated NPM level is often observed in several tumor cells such as gastric, colon, ovarian and prostate cancer, bladder, breast cancers [72-77]. Recent studies have reported that overexpression of NPM promotes cell survival, inhibits apoptosis, and induces the migration and invasion of cancer cells [78-80], supporting a role of NPM as an oncogene. Taken together, all these contradicting discoveries clearly demonstrate that NPM plays an important role in tumorigenesis, either as a tumor suppressor, an oncogene, or both.

NPM1 is one of the most frequently mutated genes in acute myeloid leukemia (AML). About $35 \%$ of AML patients carrying NPMc+, the cytoplasmic-mislocated mutant form of NPM [81]. The NPMc+ mutation is caused by an insertion of four nucleotides at the exon 12 of NPM1 gene [82]. As a result, nucleolar localization signal (NLS) which located at the $C$-terminal of wild type NPM protein is disrupted and an additional nuclear export signal (NES) is inserted at the $C$-terminal of mutant NPM protein $[83,84]$. Therefore, the mutant NPMc+ protein is localized in the cytoplasm instead of nucleoli. 
A distinct expression profile was observed in AML bearing the NPMc + mutation, raising the possible connection between NPMc+ and transcriptional regulation [85].

In our laboratory, we identified NPM and NPMc+ as novel HEXIM1-binding proteins [86]. The functional interactions between HEXIM1 and NPM/NPMc + are summarized in Figure 2. Overexpression of NPM induced the proteasome-mediated degradation of HEXIM1 [86]. Since HEXIM1 is required to form the large P-TEFb complex and block kinase activity of P-TEFb, a decrease in the level of HEXIM1 would influence the equilibrium between small and large P-TEFb complexes, leading to activation of the P-TEFb-dependent transcription (Figure 2). Using a green fluorescent protein (GFP) tagged NPMc + fusion protein, we found that NPMc+ associated with HEXIM1 and sequestered a portion of HEXIM1 in the cytoplasm [86]. As a transcription factor, HEXIM1 is present in nuclei, where it regulates RNAP II transcription and P-TEFb activity. Therefore, mislocalization of HEXIM1 in the cytoplasm would decrease the formation of the large/inactive P-TEFb complexes and thereby results in higher RNAP II transcription (Figure 2).

To determine the physiological importance of our findings, we analyzed the level and sub-cellular distribution of HEXIM1 in an AML cell line carrying the NPMc+ mutation (i.e., AML3 cell line). Compared to a wild-type NPM AML cell line, AML2, lower HEXIM1 protein level was detected in AML3 cells [87]. In addition, cytoplasmic localization of HEXIM1 was only observed in AML3 cells, but not in AML2 cells [86]. As expected, an increase in P-TEFb-mediated transcription was detected in AML3 cells [86]. Our results suggest the potential involvement of HEXIM1/P-TEFb in the tumorigenesis of AML bearing the NPMc+ mutation.

Figure 2. NPM binds to HEXIM1 and mediates the proteasome-dependent degradation of HEXIM1, which favors the release of the small P-TEFb (i.e., CDK9/cyclin T1) from the HEXIM1-containing large P-TEFb complexes. The cytoplasmic NPM mutant, NPMc + , associates and misallocates a portion of HEXIM1 in cytoplasm, resulting in decreases in the formation of large P-TEFb complexes and activation of RNAP II transcription.

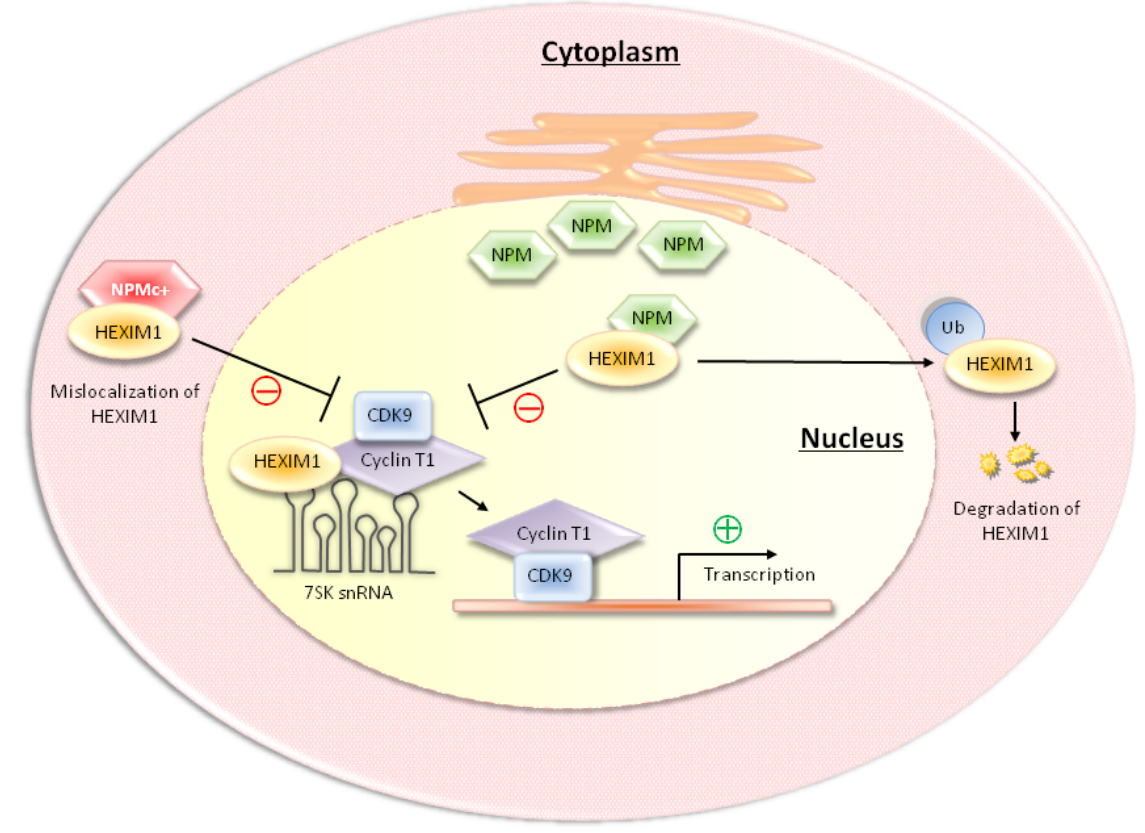




\section{HDM2 Regulates P-TEFb Activity through the Ubiquitination of HEXIM1}

MDM2 (or HDM2, the human homolog) is a well-studied negative regulator of p53 protein. The wild type p53 in unstressed cells appears to be an unstable protein with a very short half life due to MDM2-mediated proteasome degradation [56,57]. The $N$-terminus of MDM2 interacts with $N$-terminal transactivation domain of p53 and effectively blocks p53-mediated transactivation [88,89]. In addition, MDM2 carries the p53 specific E3 ubiquitin ligase within the $C$-terminal RING finger domain [57,90]. Upon interaction with p53, MDM2 E3 ligase, along with p300/CBP (CREB-binding protein) co-activator proteins, polyubiquitinates p53 in the nucleus [91-93]. This crucial step which only occurs in the nucleus would be a pre-requisite for subsequent $26 \mathrm{~S}$ proteasome degradation [94]. MDM2 RING finger domain, but not the leucine-rich nuclear export signal (NES), is important for the relocalization of p53 out of the nucleus into the cytoplasm for proteasome degradation $[95,96]$. However, 26S proteasome is present in both the nucleus and the cytoplasmic compartment [97]. In parallel to cytoplasmic compartment, the nucleus proteasome also contributed to MDM2-mediated p53 degradation pathway.

Many strategies have been formulated to disrupt the MDM2-p53 interaction as the anti-cancer approaches. Chene and co-workers designed a synthetic peptide based on the X-ray structure of p53 co-crystallizing with HDM2 [98]. When used with tumor cells that overexpress HDM2, this peptide induced the death of these tumor cells by apoptosis [98]. Nutlin-3 is a small compound that mimics the interaction of $\mathrm{p} 53$ protein and potently competes out p53 from MDM2. Importantly, treatment with nutlin-3 also stimulates a dose-dependent increase in the expression level of p21 and anti-proliferative activities across different cell lines carrying wide type p53 [99]. Similarly, another small molecule, RITA (reactivation of p53 and induction of tumor cell apoptosis), binds directly to p53. It induces a conformational change in p53 and abolishes p53-HDM2 interaction to activate p53 [100]. Additionally, the application of antibodies against MDM2 was proposed. Microinjection of an antibody specifically targeting against the p53 binding domain of MDM2 effectively disrupts the MDM2-p53 complex formation to increase p53-dependent transcription activation [101]. The ARF protein, which is introduced formerly, plays a significant role as a tumor suppressor that blocks the MDM2-dependent p53 degradation. An ARF synthetic peptide was designed to imitate the $N$-terminal domain of ARF. Acting like ARF, this synthetic peptide was shown to bind directly onto the central acidic domain of MDM2, inhibit MDM2-dependent ubiquitination and protect p53 from ubiquitination-mediated proteasome degradation [102].

Since we have established HEXIM1 to have a connection with NPM and NPM in turn also interacts with HDM2, such close associations allow us to anticipate HEXIM1 as a new substrate for HDM2. Indeed, HDM2 ubiquitinates the lysine residues located within the BR of HEXIM1; however, the ubiquitination of HEXIM1 by HDM2 does not lead to proteasome degradation pathway [103]. To investigate the impact of ubiquitination on HEXIM1's function, we generated the HEXIM1-ubiquitin fusion protein and examined its effect on $\mathrm{P}-\mathrm{TEFb}$-dependent transcription. Compared to the wild-type HEXIM1, the ubiquitinated HEXIM1 exhibited stronger inhibition on P-TEFb activity, suggesting a role of HDM2 in P-TEFb regulation [103]. On the other hand, the possible involvement of HEXIM1 in the p53 pathway is suggested. As a new binding protein and an enzymatic substrate of HDM2, HEXIM1 may have an impact on the p53 stability mediated through HDM2. It would be worthwhile to 
design a series of synthetic peptides based on the amino acid sequences of HEXIM1 BR and evaluate their effects on p53 activation.

\section{HEXIM1 Stabilizes p53 through the Protein-Protein Interaction with p53}

In the late 1980 s, several discoveries have well defined p53 to be an anti-oncogenic protein. Studies have shown that cells lacking p53 progressively become tumors during excessive unregulated genomic mutations [50,52,104]. Henceforth, stabilizing p53 has been a focus as a potential remedy for cancers [50-53,104-109]. Of the many studies on positive regulators of p53, several are relevant to our discussion of HEXIM1. For instance, in 1998, An et al. identified hypoxia-inducible factor 1alpha (HIF- $\alpha$ ) as p53 interacting counterpart. Under hypoxia condition, HIF- $\alpha$ becomes activated to stabilize and transactivate p53 [110,111]. Shortly after, Yuan and co-workers determined that the $N$-terminal region of p53 interacted with p300/CBP. Through p53-p300/CBP interaction, the p53 $C$-terminal domain gets acetylated and mediates p53 transactivation as well [112]. Herpesvirus-associated ubiquitin-specific protease (HAUSP) has been found to associate with and deubiquitinate $\mathrm{p} 53$, which is crucial for tumor suppression function [53,107]. The von Hippel-Lindau tumor suppressor protein (pVHL) was later found to directly interact with p53 and p300 acetylation ensued upon genotoxic stress. Moreover, acetylation thwarts the nuclear-export of p53 preventing it from HDM2 proteasome degradation and ultimately stabilizes p53 to execute cell arrest or apoptosis [108,112].

In our recent study, we demonstrated that HEXIM1 interacted directly with p53 protein via co-immunoprecipitation and GST pull-down assays [113]. It is through the discovery of NPM-HEXIM1 and HDM2-HEXIM1 interactions in which we chanced upon p53 to be a new partnering candidate for HEXIM1. The involvement of HEXIM1 in regulation of p53 activation is summarized in Figure 3. It is noteworthy that p53 interacts with the "free" HEXIM1 rather than the HEXIM1 present in the large inactive P-TEFb complex [113]. Domain study demonstrated that HEXIM1 BR (basic region) in cooperation with the $C$-terminus of HEXIM1 was required for sufficient p53 binding. Interestingly, we unraveled the NEG (negative regulation) domain of p53 was essential to interact with HEXIM1 [113]. With reference to Figure 4, the NEG domain consists of various lysine residues which have been well-established as the HDM2 ubiquitination sites [50-55]. Since these ubiquitination sites resided in the HEXIM1 binding domain of p53, we reckoned that HEXIM1 occupancy to the NEG domain might hinder the p53-HDM2 interaction and thereby block the ubiquitination of p53 (Figure 4). As expected, overexpression of HEXIM1 disrupts the interaction between HDM2 and p53 resulting in stabilization of p53 and activation of p53 downstream targets (such as PUMA and p21) in various cancer cell lines [113]. This finding further verify that HEXIM1 might compete with HDM2 in binding to p53 at the same site (Figure 3) [113]. Previously, we also detected the association between HDM2 and HEXIM1, resulting in ubiquitination of HEXIM1 [103]. This HEXIM1-HDM2 interaction may protect a portion of p53 from being associated with and ubiquitinated by HDM2, and indirectly contribute to the stability of p53 (Figure 3). 
Figure 3. Treatments with UV radiation, flavopiridol, DRB, roscovitine, actinomycin D, and doxorubicin, etoposide, and nutlin-3 increase the HEXIM1-p53 interaction and lead to induction of p53. UV, flavopiridol, DRB, roscovitine, and actinomycin D treatments can release more "free" HEXIM1 from the large P-TEFb complexes and may further enhance the association between p53 and HEXIM1. HEXIM1 not only competes with HDM2 in binding to p53, but also interacts with HDM2, resulting in activation of p53. The HDM2-ubiquitinated HEXIM1, which is not degraded through the proteasome-mediated pathway, exerts stronger inhibition on $\mathrm{P}-\mathrm{TEFb}$ activity.

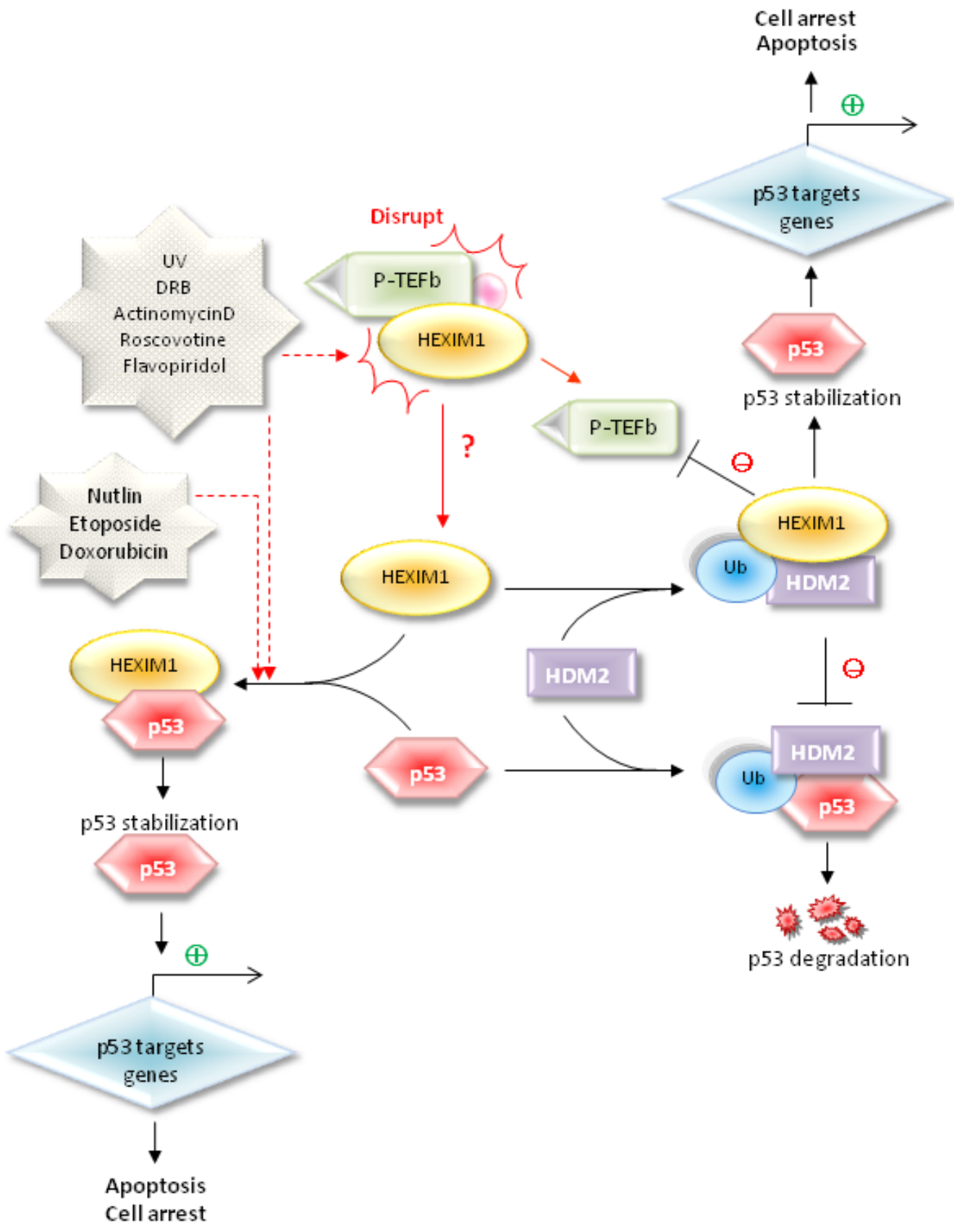


Figure 4. The NEG domain of p53 contains the lysine residues targeted by HDM2 for ubiquitination and degradation of p53. Domain study of p53 reveals that the NEG domain is essential for the association between HEXIM1 and p53 [113]. It is proposed that HEXIM1 binds and stabilizes p53 protein by blocking the ubiquitination of p53 by HDM2.

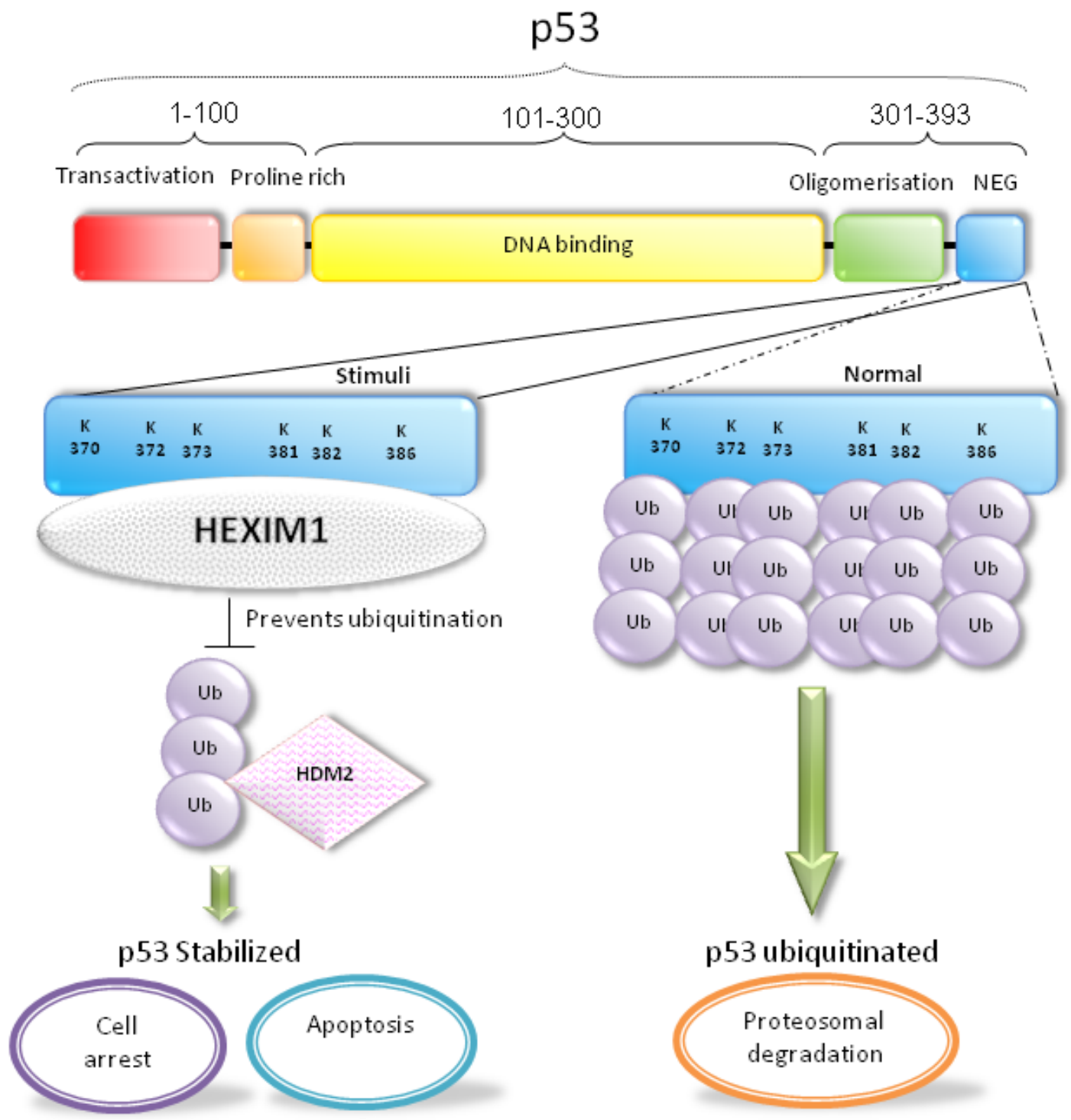

Remarkably, treatments with UV radiation, CDK inhibiting (flavopiridol, DRB, roscovitine), transcription inhibiting (actinomycin D), and p53 inducing compounds (doxorubicin, etoposide, nutlin-3) not only increase p53 levels [54,105,113], but also enhance the protein-protein interactions between HEXIM1 and p53 (Figure 3) [113]. UV, flavopiridol, DRB, roscovitine, and actinomycin D treatments disrupt the formation of large P-TEFb complexes, resulting in releasing more HEXIM1 from the large P-TEFb complexes. Such treatments should increase the pool of "free" HEXIM1 in cells and may contribute to the increased p53-HEXIM1 interaction (Figure 3). However, treatment with doxorubicin, etoposide, and nutlin-3, which has no effects on the formation of large P-TEFb complexes, enhances the p53-HEXIM1 association as well (Figure 3) [113]. This observation indicates that cells should have plenty of the "free" HEXIM1 to interact with p53 upon the stimulation [6,114]. Unlike doxorubicin and etoposide (topoisomerase II inhibitors), treatment with camptothecin, an topoisomerase I inhibitor and a 
p53 inducing compound, dissociates large P-TEFb complexes [115]. Although these three compounds affect p53 induction in a similar way, they may utilize different mechanisms to influence other biological processes. While the mechanism for the camptothecin-mediated disruption of large P-TEFb complexes remains unknown, it is worthwhile to determine the role of HEXIM1 in p53 activation induced by camptothecin. Importantly, HEXIM1 knockdown cells would not respond to treatment of p53 inducing reagents (i.e., flavopiridol and doxorubicin) and did not up-regulate the expression of p53 downstream targets. This unmistakably emphasizes the significance of HEXIM1 in p53 activation following these treatments [113].

Claudio et al. found that P-TEFb bound to the $C$-terminal domain (a.a. 361-393) of p53 and phosphorylated p53 at serine 392 [116]. In another report, Radhakrishnan and co-worker adopted the mass spectrometry technique to demonstrate that CDK9 phosphorylated p53 at Ser-33 and Ser-392 [117]. Phosphorylation of Ser33, Ser315, and Ser392 enhances the DNA binding ability and induces transactivation of p53 [118-120]. Overexpression of HEXIM1 was found to maintain the phosphorylation of p53 at Ser-33 and Ser-392, and activate the expression of p53 downstream targets, p21 and PUMA [113]. The p53-P-TEFb and p53-HEXIM1 interactions may be two independent events. It is possible that $\mathrm{P}-\mathrm{TEFb}$ may phosphorylate p53 first. After dissociating with $\mathrm{P}-\mathrm{TEFb}$, the phosphorylated p53 binds to HEXIM1, which further stabilizes the phosphorylation of p53 at Ser-33 and Ser-392. Although it has not been determined in our report whether P-TEFb actually participates in p53 phosphorylation, nevertheless, there is a likelihood that other kinases are involving [109,121,122].

\section{Conclusions}

Both p53 and P-TEFb are essential cellular regulators. Generally, p53 is involved in all adult cancers with about $50 \%$ of the cancer patients acquiring p53 mutations while the other half is due to the suppression of p53 functions [104]. P-TEFb regulates most transcription by RNAP II and its kinase activity is tightly guarded by HEXIM1. Our studies in the past few years establish the functional connection between these two important pathways. NPM and HDM2 regulate P-TEFb activity through the modulation of HEXIM1, while HEXIM1 induces p53 activation by enhancing its stability. The novel discovery of HEXIM1 in regulating p53 suggests a molecular mechanism for p53 activation induced by anti-cancer drugs/compounds through the interaction with HEXIM1. HEXIM2, a protein sharing extensive homology at the central and $C$-terminal regions of HEXIM1, exhibits similar functions in regulating P-TEFb activity $[30,32,33]$. It is noteworthy that the central and $C$-terminal domains of HEXIM1 are required for the interaction with p53 [113]. Future investigation is required to examine the involvement of HEXIM2 in the p53 pathway. These discoveries will impart better understanding on the molecular actions of p53-inducing agents which may lead to potential new strategies development for cancer therapy.

\section{Conflict of Interest}

The authors declare no conflict of interest. 


\section{References}

1. Kusuhara, M.N.K.; Kimura, K.; Maass, N.; Manabe, T.; Ishikawa, S.; Aikawa, M.; Miyazaki, K.; Yamaguchi, K. Cloning of hexamethylene-bis-acetamide-inducible transcript, HEXIM1, in human vascular smooth muscle cells. Biomed. Res. 1999, 20, 273-279.

2. Ghatpande, S.; Goswami, S.; Mathew, S.; Rong, G.; Cai, L.; Shafiq, S.; Siddiqui, M.A. Identification of a novel cardiac lineage-associated protein(cCLP-1): A candidate regulator of cardiogenesis. Dev. Biol. 1999, 208, 210-221.

3. Huang, F.; Wagner, M.; Siddiqui, M.A. Ablation of the CLP-1 gene leads to down-regulation of the HAND1 gene and abnormality of the left ventricle of the heart and fetal death. Mech. Dev. 2004, 121, 559-572.

4. Huang, F.; Wagner, M.; Siddiqui, M.A. Structure, expression, and functional characterization of the mouse CLP-1 gene. Gene 2002, 292, 245-259.

5. Wittmann, B.M.; Wang, N.; Montano, M.M. Identification of a novel inhibitor of breast cell growth that is down-regulated by estrogens and decreased in breast tumors. Cancer Res. 2003, 63, 5151-5158.

6. Michels, A.A.; Nguyen, V.T.; Fraldi, A.; Labas, V.; Edwards, M.; Bonnet, F.; Lania, L.; Bensaude, O. MAQ1 and 7SK RNA interact with CDK9/cyclin T complexes in a transcriptiondependent manner. Mol. Cell Biol. 2003, 23, 4859-4869.

7. Yik, J.H.; Chen, R.; Nishimura, R.; Jennings, J.L.; Link, A.J.; Zhou, Q. Inhibition of P-TEFb (CDK9/Cyclin T) kinase and RNA polymerase II transcription by the coordinated actions of HEXIM1 and 7SK snRNA. Mol. Cell 2003, 12, 971-982.

8. Marshall, N.F.; Price, D.H. Control of formation of two distinct classes of RNA polymerase II elongation complexes. Mol. Cell Biol. 1992, 12, 2078-2090.

9. Marshall, N.F.; Price, D.H. Purification of P-TEFb, a transcription factor required for the transition into productive elongation. J. Biol. Chem. 1995, 270, 12335-12338.

10. Peng, J.; Marshall, N.F.; Price, D.H. Identification of a cyclin subunit required for the function of Drosophila P-TEFb. J. Biol. Chem. 1998, 273, 13855-13860.

11. Peng, J.; Zhu, Y.; Milton, J.T.; Price, D.H. Identification of multiple cyclin subunits of human P-TEFb. Genes Dev. 1998, 12, 755-762.

12. Price, D.H. P-TEFb, a cyclin-dependent kinase controlling elongation by RNA polymerase II. Mol. Cell Biol. 2000, 20, 2629-2634.

13. Peterlin, B.M.; Price, D.H. Controlling the elongation phase of transcription with P-TEFb. Mol. Cell 2006, 23, 297-305.

14. Chao, S.H.; Fujinagam, K.; Marion, J.E.; Taube, R.; Sausville, A.; Senderowicz, A.M.; Peterlin, B.M.; Price, D.H. Flavopiridol inhibits P-TEFb and blocks HIV-1 replication. J. Biol. Chem. 2000, 275, 28345-28348.

15. Chao, S.H.; Price, D.H. Flavopiridol inactivates P-TEFb and blocks most RNA polymerase II transcription in vivo. J. Biol. Chem. 2001, 276, 31793-31799.

16. Guenther, M.G.; Levine, S.S.; Boyer, L.A.; Jaenisch, R.; Young, R.A. A chromatin landmark and transcription initiation at most promoters in human cells. Cell 2007, 130, 77-88. 
17. Zeitlinger, J.; Stark, A.; Kellis, M.; Hong, J.W.; Nechaev, S.; Adelman, K.; Levine, M.; Young, R.A. RNA polymerase stalling at developmental control genes in the Drosophila melanogaster embryo. Nat. Genet. 2007, 39, 1512-1516.

18. Muse, G.W.; Gilchrist, D.A.; Nechaev, S.; Shah, R.; Parker, J.S.; Grissom, S.F.; Zeitlinger, J.; Adelman, K. RNA polymerase is poised for activation across the genome. Nat. Genet. 2007, 39, $1507-1511$.

19. Wimmer, J.; Fujinaga, K.; Taube, R.; Cujec, T.P.; Zhu, Y.; Peng, J.; Price, D.H.; Peterlin, B.M. Interactions between Tat and TAR and human immunodeficiency virus replication are facilitated by human cyclin T1 but not cyclins T2a or T2b. Virology 1999, 255, 182-189.

20. Wei, P.; Garber, M.E.; Fang, S.M.; Fischer, W.H.; Jones, K.A. A novel CDK9-associated C-type cyclin interacts directly with HIV-1 Tat and mediates its high-affinity, loop-specific binding to TAR RNA. Cell 1998, 92, 451-462.

21. Zhu, Y.; Peery, T.; Peng, J.; Ramanathan, Y.; Marshall, N.; Marshall, T.; Amendt, B.; Mathews, M.B.; Price, D.H. Transcription elongation factor P-TEFb is required for HIV-1 tat transactivation in vitro. Genes Dev. 1997, 11, 2622-2632.

22. Ramanathan, Y.; Reza, S.M.; Young, T.M.; Mathews, M.B.; Peery, T. Human and rodent transcription elongation factor P-TEFb: Interactions with human immunodeficiency virus type 1 tat and carboxy-terminal domain substrate. J. Virol. 1999, 73, 5448-5458.

23. Garriga, J.; Mayol, X.; Grana, X. The CDC2-related kinase PITALRE is the catalytic subunit of active multimeric protein complexes. Biochem. J. 1996, 319, 293-298.

24. Nguyen, V.T.; Kiss, T.; Michels, A.A.; Bensaude, O. 7SK small nuclear RNA binds to and inhibits the activity of CDK9/cyclin T complexes. Nature 2001, 414, 322-325.

25. Yang, Z.; Zhu, Q.; Luo, K.; Zhou, Q. The 7SK small nuclear RNA inhibits the CDK9/cyclin T1 kinase to control transcription. Nature 2001, 414, 317-322.

26. Michels, A.A.; Fraldi, A.; Li, Q.; Adamson, T.E.; Bonnet, F.; Nguyen, V.T.; Sedore, S.C.; Price, J.P.; Price, D.H.; Lania, L.; et al. Binding of the 7SK snRNA turns the HEXIM1 protein into a P-TEFb (CDK9/cyclin T) inhibitor. EMBO J. 2004, 23, 2608-2619.

27. Krueger, B.J.; Jeronimo, C.; Roy, B.B.; Bouchard, A.; Barrandon, C.; Byers, S.A.; Searcey, C.E.; Cooper, J.J.; Bensaude, O.; Cohen, E.A.; et al. LARP7 is a stable component of the 7SK snRNP while P-TEFb, HEXIM1 and hnRNP A1 are reversibly associated. Nucleic Acids Res. 2008, 36, 2219-2229.

28. Jeronimo, C.; Forget, D.; Bouchard, A.; Li, Q.; Chua, G.; Poitras, C.; Therien, C.; Bergeron, D.; Bourassa, S.; Greenblatt, J.; et al. Systematic analysis of the protein interaction network for the human transcription machinery reveals the identity of the 7SK capping enzyme. Mol. Cell 2007, 27, 262-274.

29. Xue, Y.; Yang, Z.; Chen, R.; Zhou, Q. A capping-independent function of MePCE in stabilizing 7SK snRNA and facilitating the assembly of 7SK snRNP. Nucleic Acids Res. 2010, 38, 360-369.

30. Li, Q.; Price, J.P.; Byers, S.A.; Cheng, D.; Peng, J.; Price, D.H. Analysis of the large inactive P-TEFb complex indicates that it contains one 7SK molecule, a dimer of HEXIM1 or HEXIM2, and two P-TEFb molecules containing Cdk9 phosphorylated at threonine 186. J. Biol. Chem. 2005, 280, 28819-28826. 
31. Barboric, M.; Kohoutek, J.; Price, J.P.; Blazek, D.; Price, D.H.; Peterlin, B.M. Interplay between 7SK snRNA and oppositely charged regions in HEXIM1 direct the inhibition of P-TEFb. EMBO J. 2005, 24, 4291-4303.

32. Blazek, D.; Barboric, M.; Kohoutek, J.; Oven, I.; Peterlin, B.M. Oligomerization of HEXIM1 via 7SK snRNA and coiled-coil region directs the inhibition of P-TEFb. Nucleic Acids Res. 2005, 33, 7000-7010.

33. Dulac, C.; Michels, A.A.; Fraldi, A.; Bonnet, F.; Nguyen, V.T.; Napolitano, G.; Lania, L.; Bensaude, O. Transcription-dependent association of multiple positive transcription elongation factor units to a HEXIM multimer. J. Biol. Chem. 2005, 280, 30619-30629.

34. Galatioto, J.; Mascareno, E.; Siddiqui, M.A. CLP-1 associates with MyoD and HDAC to restore skeletal muscle cell regeneration. J. Cell Sci. 2010, 123, 3789-3795.

35. Czudnochowski, N.; Vollmuth, F.; Baumann, S.; Vogel-Bachmayr, K.; Geyer, M. Specificity of Hexim1 and Hexim2 complex formation with cyclin T1/T2, importin alpha and 7SK snRNA. J. Mol. Biol. 2010, 395, 28-41.

36. Ali, S.; Coombes, R.C. Estrogen receptor alpha in human breast cancer: Occurrence and significance. J. Mammary Gland Biol. Neoplasia 2000, 5, 271-281.

37. Katzenellenbogen, B.S.; Frasor, J. Therapeutic targeting in the estrogen receptor hormonal pathway. Semin. Oncol. 2004, 31, 28-38.

38. Wittmann, B.M.; Fujinaga, K.; Deng, H.; Ogba, N.; Montano, M.M. The breast cell growth inhibitor, estrogen down regulated gene 1, modulates a novel functional interaction between estrogen receptor alpha and transcriptional elongation factor cyclin T1. Oncogene 2005, 24, 5576-5588.

39. Ouchida, R.; Kusuhara, M.; Shimizu, N.; Hisada, T.; Makino, Y.; Morimoto, C.; Handa, H.; Ohsuzu, F.; Tanaka, H. Suppression of NF-kappaB-dependent gene expression by a hexamethylene bisacetamide-inducible protein HEXIM1 in human vascular smooth muscle cells. Genes Cells 2003, 8, 95-107.

40. Barboric, M.; Nissen, R.M.; Kanazawa, S.; Jabrane-Ferrat, N.; Peterlin, B.M. NF-kappaB binds $\mathrm{P}-\mathrm{TEFb}$ to stimulate transcriptional elongation by RNA polymerase II. Mol. Cell 2001, 8, 327-337.

41. Shimizu, N.; Ouchida, R.; Yoshikawa, N.; Hisada, T.; Watanabe, H.; Okamoto, K.; Kusuhara, M.; Handa, H.; Morimoto, C.; Tanaka, H. HEXIM1 forms a transcriptionally abortive complex with glucocorticoid receptor without involving 7SK RNA and positive transcription elongation factor $b$. Proc. Natl. Acad. Sci. USA 2005, 102, 8555-8560.

42. Lane, D.P. Cancer. p53, guardian of the genome. Nature 1992, 358, 15-16.

43. May, P.; May, E. Twenty years of p53 research: Structural and functional aspects of the p53 protein. Oncogene 1999, 18, 7621-7636.

44. Lu, H.; Levine, A.J. Human TAFII31 protein is a transcriptional coactivator of the p53 protein. Proc. Natl. Acad. Sci. USA 1995, 92, 5154-5158.

45. Thut, C.J.; Chen, J.L.; Klemm, R.; Tjian, R. p53 transcriptional activation mediated by coactivators TAFII40 and TAFII60. Science 1995, 267, 100-104.

46. Sakamuro, D.; Sabbatini, P.; White, E.; Prendergast, G.C. The polyproline region of p53 is required to activate apoptosis but not growth arrest. Oncogene 1997, 15, 887-898. 
47. Walker, K.K.; Levine, A.J. Identification of a novel p53 functional domain that is necessary for efficient growth suppression. Proc. Natl. Acad. Sci. USA 1996, 93, 15335-15340.

48. Pavletich, N.P.; Chambers, K.A.; Pabo, C.O. The DNA-binding domain of p53 contains the four conserved regions and the major mutation hot spots. Genes Dev. 1993, 7, 2556-2564.

49. Chene, P. The role of tetramerization in p53 function. Oncogene 2001, 20, 2611-2617.

50. Vogelstein, B.; Lane, D.; Levine, A.J. Surfing the p53 network. Nature 2000, 408, 307-310.

51. Li, M.; Luo, J.; Brooks, C.L.; Gu, W. Acetylation of p53 inhibits its ubiquitination by Mdm2. J. Biol. Chem. 2002, 277, 50607-50611.

52. Ko, L.J.; Prives, C. p53: Puzzle and paradigm. Genes Dev. 1996, 10, 1054-1072.

53. Brooks, C.L.; Li, M.; Hu, M.; Shi, Y.; Gu, W. The p53-Mdm2-HAUSP complex is involved in p53 stabilization by HAUSP. Oncogene 2007, 26, 7262-7266.

54. Rodriguez, M.S.; Desterro, J.M.; Lain, S.; Lane, D.P.; Hay, R.T. Multiple $C$-terminal lysine residues target p53 for ubiquitin-proteasome-mediated degradation. Mol. Cell Biol. 2000, 20, 8458-8467.

55. Poyurovsky, M.V.; Katz, C.; Laptenko, O.; Beckerman, R.; Lokshin, M.; Ahn, J.; Byeon, I.J.; Gabizon, R.; Mattia, M.; Zupnick, A.; et al. The $C$-terminus of p53 binds the $N$-terminal domain of MDM2. Nat. Struct. Mol. Biol. 2010, 17, 982-989.

56. Levine, A.J. p53, the cellular gatekeeper for growth and division. Cell 1997, 88, 323-331.

57. Honda, R.; Tanaka, H.; Yasuda, H. Oncoprotein MDM2 is a ubiquitin ligase E3 for tumor suppressor p53. FEBS Lett. 1997, 420, 25-27.

58. Zhang, Y.; Xiong, Y.; Yarbrough, W.G. ARF promotes MDM2 degradation and stabilizes p53: ARF-INK4a locus deletion impairs both the Rb and p53 tumor suppression pathways. Cell 1998, 92, 725-734.

59. Kuo, M.L.; den Besten, W.; Bertwistle, D.; Roussel, M.F.; Sherr, C.J. N-terminal polyubiquitination and degradation of the Arf tumor suppressor. Genes Dev. 2004, 18, 1862-1874.

60. Bertwistle, D.; Sugimoto, M.; Sherr, C.J. Physical and functional interactions of the Arf tumor suppressor protein with nucleophosmin/B23. Mol. Cell Biol. 2004, 24, 985-996.

61. Kurki, S.; Peltonen, K.; Latonen, L.; Kiviharju, T.M.; Ojala, P.M.; Meek, D.; Laiho, M. Nucleolar protein NPM interacts with HDM2 and protects tumor suppressor protein p53 from HDM2-mediated degradation. Cancer Cell 2004, 5, 465-475.

62. Savkur, R.S.; Olson, M.O. Preferential cleavage in pre-ribosomal RNA byprotein B23 endoribonuclease. Nucleic Acids Res. 1998, 26, 4508-4515.

63. Itahana, K.; Bhat, K.P.; Jin, A.; Itahana, Y.; Hawke, D.; Kobayashi, R.; Zhang, Y. Tumor suppressor ARF degrades B23, a nucleolar protein involved in ribosome biogenesis and cell proliferation. Mol. Cell 2003, 12, 1151-1164.

64. Grisendi, S.; Mecucci, C.; Falini, B.; Pandolfi, P.P. Nucleophosmin and cancer. Nat. Rev. Cancer 2006, 6, 493-505.

65. Okuwaki, M.; Matsumoto, K.; Tsujimoto, M.; Nagata, K. Function of nucleophosmin/B23, a nucleolar acidic protein, as a histone chaperone. FEBS Lett. 2001, 506, 272-276.

66. Krause, A. Hoffmann I: Polo-like kinase 2-dependent phosphorylation of NPM/B23 on serine 4 triggers centriole duplication. PLoS One 2010, 5, e9849. 
67. Weber, J.D.; Taylor, L.J.; Roussel, M.F.; Sherr, C.J.; Bar-Sagi, D. Nucleolar Arf sequesters Mdm2 and activates p53. Nat. Cell Biol. 1999, 1, 20-26.

68. Kamijo, T.; Zindy, F.; Roussel, M.F.; Quelle, D.E.; Downing, J.R.; Ashmun, R.A.; Grosveld, G.; Sherr, C.J. Tumor suppression at the mouse INK4a locus mediated by the alternative reading frame product p19ARF. Cell 1997, 91, 649-659.

69. Yun, J.P.; Chew, E.C.; Liew, C.T.; Chan, J.Y.; Jin, M.L.; Ding, M.X.; Fai, Y.H.; Li, H.K.; Liang, X.M.; Wu, Q.L. Nucleophosmin/B23 is a proliferate shuttle protein associated with nuclear matrix. J. Cell Biochem. 2003, 90, 1140-1148.

70. Colombo, E.; Marine, J.C.; Danovi, D.; Falini, B.; Pelicci, P.G. Nucleophosmin regulates the stability and transcriptional activity of p53. Nat. Cell Biol. 2002, 4, 529-533.

71. Grisendi, S.; Bernardi, R.; Rossi, M.; Cheng, K.; Khandker, L.; Manova, K.; Pandolfi, P.P. Role of nucleophosmin in embryonic development and tumorigenesis. Nature 2005, 437, 147-153.

72. Tanaka, M.; Sasaki, H.; Kino, I.; Sugimura, T.; Terada, M. Genes preferentially expressed in embryo stomach are predominantly expressed in gastric cancer. Cancer Res. 1992, 52, 3372-3377.

73. Nozawa, Y.; van Belzen, N.; van der Made, A.C.; Dinjens, W.N.; Bosman, F.T. Expression of nucleophosmin/B23 in normal and neoplastic colorectal mucosa. J. Pathol. 1996, 178, 48-52.

74. Shields, L.B.; Gercel-Taylor, C.; Yashar, C.M.; Wan, T.C.; Katsanis, W.A.; Spinnato, J.A.; Taylor, D.D. Induction of immune responses to ovarian tumor antigens by multiparity. J. Soc. Gynecol. Investig. 1997, 4, 298-304.

75. Subong, E.N.; Shue, M.J.; Epstein, J.I.; Briggman, J.V.; Chan, P.K.; Partin, A.W. Monoclonal antibody to prostate cancer nuclear matrix protein (PRO:4-216) recognizes nucleophosmin/B23. Prostate 1999, 39, 298-304.

76. Tsui, K.H.; Cheng, A.J.; Chang, P.; Pan, T.L.; Yung, B.Y. Association of nucleophosmin/B23 mRNA expression with clinical outcome in patients with bladder carcinoma. Urology 2004, 64, 839-844.

77. Skaar, T.C.; Prasad, S.C.; Sharareh, S.; Lippman, M.E.; Brunner, N.; Clarke, R. Two-dimensional gel electrophoresis analyses identify nucleophosmin as an estrogen regulated protein associated with acquired estrogen-independence in human breast cancer cells. J. Steroid Biochem. Mol. Biol. 1998, 67, 391-402.

78. Li, J.; Sejas, D.P.; Rani, R.; Koretsky, T.; Bagby, G.C.; Pang, Q. Nucleophosmin regulates cell cycle progression and stress response in hematopoietic stem/progenitor cells. J. Biol. Chem. 2006, 281, 16536-16545.

79. Liu, Y.; Zhang, F.; Zhang, X.F.; Qi, L.S.; Yang, L.; Guo, H.; Zhang, N. Expression of nucleophosmin/NPM1 correlates with migration and invasiveness of colon cancer cells. J. Biomed. Sci. 2012, 19, 53.

80. Ye, K. Nucleophosmin/B23, a multifunctional protein that can regulate apoptosis. Cancer Biol. Ther. 2005, 4, 918-923.

81. Falini, B.; Mecucci, C.; Tiacci, E.; Alcalay, M.; Rosati, R.; Pasqualucci, L.; La Starza, R.; Diverio, D.; Colombo, E.; Santucci, A.; et al. Cytoplasmic nucleophosmin in acute myelogenous leukemia with a normal karyotype. N. Engl. J. Med. 2005, 352, 254-266. 
82. Quentmeier, H.; Martelli, M.P.; Dirks, W.G.; Bolli, N.; Liso, A.; Macleod, R.A.; Nicoletti, I.; Mannucci, R.; Pucciarini, A.; Bigerna, B.; et al. Cell line OCI/AML3 bears exon-12 NPM gene mutation-A and cytoplasmic expression of nucleophosmin. Leukemia 2005, 19, 1760-1767.

83. Falini, B.; Bolli, N.; Shan, J.; Martelli, M.P.; Liso, A.; Pucciarini, A.; Bigerna, B.; Pasqualucci, L.; Mannucci, R.; Rosati, R.; et al. Both carboxy-terminus NES motif and mutated tryptophan(s) are crucial for aberrant nuclear export of nucleophosmin leukemic mutants in NPMc+ AML. Blood 2006, 107, 4514-4523.

84. Falini, B.; Nicoletti, I.; Martelli, M.F.; Mecucci, C. Acute myeloid leukemia carrying cytoplasmic/mutated nucleophosmin (NPMc+ AML): Biologic and clinical features. Blood 2007, 109, 874-885.

85. Alcalay, M.; Tiacci, E.; Bergomas, R.; Bigerna, B.; Venturini, E.; Minardi, S.P.; Meani, N.; Diverio, D.; Bernard, L.; Tizzoni, L.; et al. Acute myeloid leukemia bearing cytoplasmic nucleophosmin (NPMc+ AML) shows a distinct gene expression profile characterized by up-regulation of genes involved in stem-cell maintenance. Blood 2005, 106, 899-902.

86. Gurumurthy, M.; Tan, C.H.; Ng, R.; Zeiger, L,.; Lau, J.; Lee, J.; Dey, A.; Philp, R.; Li, Q.; Lim, T.M.; et al. Nucleophosmin interacts with HEXIM1 and regulates RNA polymerase II transcription. J. Mol. Biol. 2008, 378, 302-317.

87. Lew, Q.J.; Tan, C.H.; Gurumurthy, M.; Chu, K.L.; Cheong, N.; Lane, D.P.; Chao, S.H. NPMc(+) AML cell line shows differential protein expression and lower sensitivity to DNA-damaging and p53-inducing anticancer compounds. Cell Cycle 2011, 10, 1978-1987.

88. Momand, J.; Zambetti, G.P.; Olson, D.C.; George, D.; Levine, A.J. The mdm-2 oncogene product forms a complex with the p53 protein and inhibits p53-mediated transactivation. Cell 1992, 69, 1237-1245.

89. Oliner, J.D.; Pietenpol, J.A.; Thiagalingam, S.; Gyuris, J.; Kinzler, K.W.; Vogelstein, B. Oncoprotein MDM2 conceals the activation domain of tumour suppressor p53. Nature 1993, $362,857-860$.

90. Fang, S.; Jensen, J.P.; Ludwig, R.L.; Vousden, K.H.; Weissman, A.M. Mdm2 is a RING finger-dependent ubiquitin protein ligase for itself and p53. J. Biol. Chem. 2000, 275, 8945-8951.

91. Lai, Z.; Ferry, K.V.; Diamond, M.A.; Wee, K.E.; Kim, Y.B.; Ma, J.; Yang, T.; Benfield, P.A.; Copeland, R.A.; Auger, K.R. Human mdm2 mediates multiple mono-ubiquitination of p53 by a mechanism requiring enzyme isomerization. J. Biol. Chem. 2001, 276, 31357-31367.

92. Grossman, S.R.; Deato, M.E.; Brignone, C.; Chan, H.M.; Kung, A.L.; Tagami, H.; Nakatani, Y.; Livingston, D.M. Polyubiquitination of p53 by a ubiquitin ligase activity of p300. Science 2003, 300, 342-344.

93. Ito, A.; Lai, C.H.; Zhao, X.; Saito, S.; Hamilton, M.H.; Appella, E.; Yao, T.P. p300/CBP-mediated p53 acetylation is commonly induced by p53-activating agents and inhibited by MDM2. EMBO J. 2001, 20, 1331-1340.

94. Yu, Z.K.; Geyer, R.K.; Maki, C.G. MDM2-dependent ubiquitination of nuclear and cytoplasmic P53. Oncogene 2000, 19, 5892-5897.

95. Geyer, R.K.; Yu, Z.K.; Maki, C.G. The MDM2 RING-finger domain is required to promote p53 nuclear export. Nat. Cell Biol. 2000, 2, 569-573. 
96. Boyd, S.D.; Tsai, K.Y.; Jacks, T. An intact HDM2 RING-finger domain is required for nuclear exclusion of p53. Nat. Cell Biol. 2000, 2, 563-568.

97. Palmer, A.; Mason, G.G.; Paramio, J.M.; Knecht, E.; Rivett, A.J. Changes in proteasome localization during the cell cycle. Eur. J. Cell Biol. 1994, 64, 163-175.

98. Chene, P.; Fuchs, J.; Bohn, J.; Garcia-Echeverria, C.; Furet, P.; Fabbro, D. A small synthetic peptide, which inhibits the p53-hdm2 interaction, stimulates the p53 pathway in tumour cell lines. J. Mol. Biol. 2000, 299, 245-253.

99. Vassilev, L.T.; Vu, B.T.; Graves, B.; Carvajal, D.; Podlaski, F.; Filipovic, Z.; Kong, N.; Kammlott, U.; Lukacs, C.; Klein, C.; et al. In vivo activation of the p53 pathway by small-molecule antagonists of MDM2. Science 2004, 303, 844-848.

100. Issaeva, N.; Bozko, P.; Enge, M.; Protopopova, M.; Verhoef, L.G.; Masucci, M.; Pramanik, A.; Selivanova, G. Small molecule RITA binds to p53, blocks p53-HDM-2 interaction and activates p53 function in tumors. Nat. Med. 2004, 10, 1321-1328.

101. Blaydes, J.P.; Gire, V.; Rowson, J.M.; Wynford-Thomas, D. Tolerance of high levels of wild-type p53 in transformed epithelial cells dependent on auto-regulation by mdm-2. Oncogene 1997, 14, 1859-1868.

102. Midgley, C.A.; Desterro, J.M.; Saville, M.K.; Howard, S.; Sparks, A.; Hay, R.T.; Lane, D.P. An $N$-terminal p14ARF peptide blocks Mdm2-dependent ubiquitination in vitro and can activate p53 in vivo. Oncogene 2000, 19, 2312-2323.

103. Lau, J.; Lew, Q.J.; Diribarne, G.; Michels, A.A.; Dey, A.; Bensaude, O.; Lane, D.P.; Chao, S.H. Ubiquitination of HEXIM1 by HDM2. Cell Cycle 2009, 8, 2247-2254.

104. Brown, C.J.; Lain, S.; Verma, C.S.; Fersht, A.R.; Lane, D.P. Awakening guardian angels: Drugging the p53 pathway. Nat. Rev. Cancer 2009, 9, 862-873.

105. Fritsche, M.; Haessler, C.; Brandner, G. Induction of nuclear accumulation of the tumor-suppressor protein p53 by DNA-damaging agents. Oncogene 1993, 8, 307-318.

106. El-Deiry, W.S.; Tokino, T.; Velculescu, V.E.; Levy, D.B.; Parsons, R.; Trent, J.M.; Lin, D.; Mercer, W.E.; Kinzler, K.W.; Vogelstein, B. WAF1, a potential mediator of p53 tumor suppression. Cell 1993, 75, 817-825.

107. Cummins, J.M.; Vogelstein, B. HAUSP is required for p53 destabilization. Cell Cycle 2004, 3, 689-692.

108. Roe, J.S.; Kim, H.; Lee, S.M.; Kim, S.T.; Cho, E.J.; Youn, H.D. p53 stabilization and transactivation by a von Hippel-Lindau protein. Mol. Cell 2006, 22, 395-405.

109. Thompson, T.; Tovar, C.; Yang, H.; Carvajal, D.; Vu, B.T.; Xu, Q.; Wahl, G.M.; Heimbrook, D.C.; Vassilev, L.T. Phosphorylation of p53 on key serines is dispensable for transcriptional activation and apoptosis. J. Biol. Chem. 2004, 279, 53015-53022.

110. An, W.G.; Kanekal, M.; Simon, M.C.; Maltepe, E.; Blagosklonny, M.V.; Neckers, L.M. Stabilization of wild-type p53 by hypoxia-inducible factor 1alpha. Nature 1998, 392, 405-408.

111. Hammond, E.M.; Giaccia, A.J. Hypoxia-inducible factor-1 and p53: Friends, acquaintances, or strangers? Clin. Cancer Res. 2006, 12, 5007-5009.

112. Yuan, Z.M.; Huang, Y.; Ishiko, T.; Nakada, S.; Utsugisawa, T.; Shioya, H.; Utsugisawa, Y.; Yokoyama, K.; Weichselbaum, R.; Shi, Y.; et al. Role for p300 in stabilization of p53 in the response to DNA damage. J. Biol. Chem. 1999, 274, 1883-1886. 
113. Lew, Q.J.; Chia, Y.L.; Chu, K.L.; Lam, Y.T.; Gurumurthy, M.; Xu, S.; Lam, K.P.; Cheong, N.; Chao, S.H. Identification of HEXIM1 as a Positive Regulator of p53. J. Biol. Chem. 2012, 287, 36443-36454.

114. Byers, S.A.; Price, J.P.; Cooper, J.J.; Li, Q.; Price, D.H. HEXIM2, a HEXIM1-related protein, regulates positive transcription elongation factor $\mathrm{b}$ through association with $7 \mathrm{SK}$. J. Biol. Chem. 2005, 280, 16360-16367.

115. Amente, S.; Gargano, B.; Napolitano, G.; Lania, L.; Majello, B. Camptothecin releases P-TEFb from the inactive 7SK snRNP complex. Cell Cycle 2009, 8, 1249-1255.

116. Claudio, P.P.; Cui, J.; Ghafouri, M.; Mariano, C.; White, M.K.; Safak, M.; Sheffield, J.B.; Giordano, A.; Khalili, K.; Amini, S.; et al. Cdk9 phosphorylates p53 on serine 392 independently of CKII. J. Cell Physiol. 2006, 208, 602-612.

117. Radhakrishnan, S.K.; Gartel, A.L. CDK9 phosphorylates p53 on serine residues 33, 315 and 392. Cell Cycle 2006, 5, 519-521.

118. Zheng, H.; You, H.; Zhou, X.Z.; Murray, S.A.; Uchida, T.; Wulf, G.; Gu, L.; Tang, X.; Lu, K.P.; Xiao, Z.X. The prolyl isomerase Pin1 is a regulator of p53 in genotoxic response. Nature 2002, 419, 849-853.

119. Zacchi, P.; Gostissa, M.; Uchida, T.; Salvagno, C.; Avolio, F.; Volinia, S.; Ronai, Z.; Blandino, G.; Schneider, C.; del Sal, G. The prolyl isomerase Pin1 reveals a mechanism to control p53 functions after genotoxic insults. Nature 2002, 419, 853-857.

120. Hupp, T.R.; Meek, D.W.; Midgley, C.A.; Lane, D.P. Regulation of the specific DNA binding function of p53. Cell 1992, 71, 875-886.

121. Jeong, J.H.; Nakajima, H.; Magae, J.; Furukawa, C.; Taki, K.; Otsuka, K.; Tomita, M.; Lee, I.S.; Kim, C.H.; Chang, H.W.; et al. Ascochlorin activates p53 in a manner distinct from DNA damaging agents. Int. J. Cancer 2009, 124, 2797-2803.

122. Keller, D.M.; Zeng, X.; Wang, Y.; Zhang, Q.H.; Kapoor, M.; Shu, H.; Goodman, R.; Lozano, G.; Zhao, Y.; Lu, H. A DNA damage-induced p53 serine 392 kinase complex contains CK2, hSpt16, and SSRP1. Mol. Cell 2001, 7, 283-292.

(C) 2013 by the authors; licensee MDPI, Basel, Switzerland. This article is an open access article distributed under the terms and conditions of the Creative Commons Attribution license (http://creativecommons.org/licenses/by/3.0/). 